\title{
Regioselective synthesis and evaluation of 3-alkylidene-1, 3-dihydroisobenzofurans as potential antidepressant agents
}

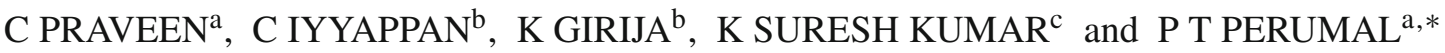 \\ ${ }^{a}$ Organic Chemistry Division, Central Leather Research Institute, Adyar, Chennai 600 020, India \\ ${ }^{b}$ Department of Pharmaceutical Chemistry, Mother Theresa Post Graduate and Research Institute of Health \\ Sciences, Puducherry 605 006, India \\ ${ }^{\mathrm{c} B i o i n f o r m a t i c s ~ C e n t r e, ~ P o n d i c h e r r y ~ U n i v e r s i t y, ~ P u d u c h e r r y ~} 605$ 014, India \\ e-mail: ptperumal@gmail.com
}

MS received 24 January 2011; revised 26 May 2011; accepted 30 May 2011

\begin{abstract}
Alkylidene-1,3-dihydroisobenzofurans exhibited moderate antidepressant activity as evaluated by forced swim and tail suspension test methods. Virtual screening was carried out by docking the designed compounds into the serotonin binding sites of arabinase protein to predict the analogue binding mode of the compounds to the SSRIs.
\end{abstract}

Keywords. Phthalans; regioselectivity; antidepressant; tail suspension test; forced swim test; molecular docking.

\section{Introduction}

Depression is a serious disorder with estimates of lifetime prevalence as high as $21 \%$ of the general population in some developed countries. ${ }^{1}$ Treatment for this disease is possible with antidepressant medications and psychotherapy for certain patients. ${ }^{2}$ Tricyclics, monoamine oxidase (MAO) inhibitors, selective serotonin reuptake inhibitors, serotonin-norepinephrine reuptake inhibitors, norepinephrine-dopamine reuptake inhibitors, norepinephrine reuptake inhibitors, serotonin modulators and norepinephrine-serotonin modulators are the major antidepressant drug classes used for the treatment of depressive disorders. ${ }^{3}$ Although antidepressants have been used in the clinic for several decades, most of them are inadequate in efficiency and have many adverse side effects. ${ }^{4}$ Therefore, studies for discovering and developing new antidepressant drugs with greater effectiveness and lower adverse effects are still desirable. ${ }^{5,6}$ The introduction of selective serotonin reuptake inhibitors (SSRIs) as second generation antidepressants was a breakthrough in the treatment of depression. The SSRIs are characterized by significantly reduced disadvantageous properties. These drugs produce notably fewer adverse drug reactions (ADRs) compared with older drugs such as tricyclic antidepressants (TCAs) or non-selective monoamine oxidase inhibitors (MAOIs). However, SSRIs also have several drawbacks including lower clinical effectiveness, ADRs and relatively long onset of action which led to the idea to develop newer, more efficacious and safer antidepressants. ${ }^{7-9}$ In view of getting broad spectrum of activity with more potency, a series of 3- alkylidene-1,3-dihydroisobenzofurans have been synthesized and screened for their antidepressant activity. These series comprise the derivatized phthalan (1,3dihydroisobenzofuran) pharmacophore that are structurally related to the standard antidepressant drug, citalopram (SSRIs). The thrust of efforts in the derivatization of such type of compounds focused mainly on the aryl moiety as well as on dihydrobenzofuran scaffold. In the present study, the substitution pattern on the aryl moiety and dihydroisobenzofuran pharmacophore was selected so as to confer a different electronic environment that would affect the lipophilicity and hence the affinity of the target molecules (figure 1).

The objective of forming these hybrids is an attempt to reach an active antidepressant agent with potential activity and selectivity towards serotonin (5-hydroxytryptamine). Moreover, drug-likeness and molecular docking were used to identify the structural features required for antidepressant properties of these new series of phthalan derivatives. The results of docking studies could support the postulation that the more active compounds may act on the same receptor target, where the animal study conformed the

*For correspondence 


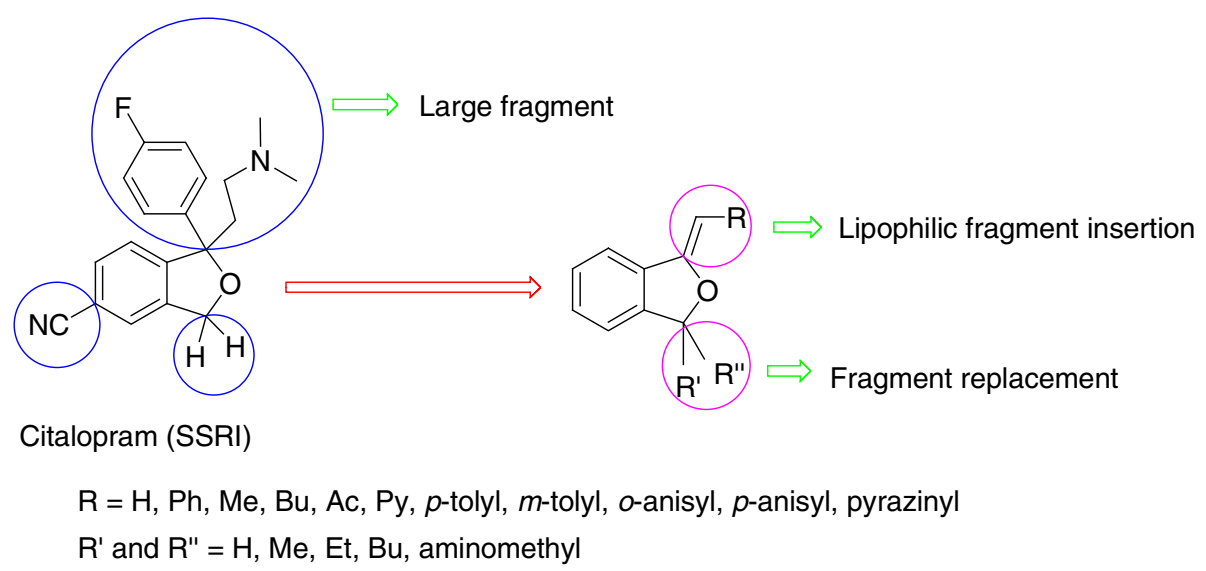

Figure 1. Reported and proposed antidepressant (SSRI) phthalan derivatives.

molecular design of the reported class of antidepressant agents.

\section{Experimental}

\subsection{Materials, method and instruments}

Melting points were determined in capillary tubes and are uncorrected. IR spectra were taken as neat for liquid compounds and as $\mathrm{KBr}$ pellets for solids on a Perkin Elmer Spectrum RXI FT-IR. ${ }^{1} \mathrm{H}$ NMR $(500 \mathrm{MHz})$ and ${ }^{13} \mathrm{C}$ NMR $(125 \mathrm{MHz})$ spectra were recorded as $\mathrm{CDCl}_{3}$ solution for compounds $\mathbf{2 a}-\mathbf{i}, \mathbf{2 n}$ and $\mathbf{2 o}$ and as benzene- $d_{6}$ solution for compounds $\mathbf{2} \mathbf{j}-\mathbf{m}$ on a JEOL instrument. Proton chemical shifts $(\delta)$ are relative to tetramethylsilane (TMS, $\delta=0.00$ ) as internal standard and expressed in parts per million. Spin multiplicities are given as $\mathrm{s}$ (singlet), $\mathrm{d}$ (doublet), $\mathrm{t}$ (triplet) and $\mathrm{m}$ (multiplet). Coupling constants $(J)$ are given in hertz. Mass spectra were recorded on a Thermo Finnigan LCQ Advantage MAX 6000 ESI spectrometer. Elemental analysis data were recorded using Thermo Finnigan FLASH EA $1112 \mathrm{CHN}$ analyzer. All the compounds gave $\mathrm{C}, \mathrm{H}$ and $\mathrm{N}$ analysis within $\pm 0.5 \%$ of the theoretical values. Column chromatography was performed on silica gel (100-200 mesh, SRL, India). Analytical TLC was performed on precoated plastic sheets of silica gel G/UV-254 of $0.2 \mathrm{~mm}$ thickness (Macherey-Nagel, Germany) using analytical grade solvents and visualizing with iodine spray $(10 \%(\mathrm{w} / \mathrm{w})$ $\mathrm{I}_{2}$ in silica gel or UV light $(\lambda=254$ and $365 \mathrm{~nm})$. UV-Visible spectra were recorded on THERMAL spectrophotometer. 2-(Ethynyl)benzyl alcohols were prepared by the Sonagashira reaction of 2-iodobenzyl alcohol with the corresponding terminal alkyne. Copper(II) trifluoromethanesulphonate was purchased from Sigma-Aldrich Ltd.

\subsection{General procedure for the synthesis of compounds (2a-0)}

2.2a Representative procedure for the synthesis of compounds (2a-i, 2n and 2o): To a degassed solution of (5-(2-phenylethynyl)benzo[d][1,3]dioxol-6-yl) methanol (1i, $252 \mathrm{mg}, 1.0 \mathrm{mmol})$ in dry toluene $(1 \mathrm{~mL})$ under $\mathrm{N}_{2}$ was added $\mathrm{Cu}(\mathrm{OTf})_{2}(18.08 \mathrm{mg}, 0.018 \mathrm{mmol})$ and the reaction mixture was stirred at $110^{\circ} \mathrm{C}$ for $30 \mathrm{~min}$. After completion of the reaction as indicated by TLC, the reaction mixture was concentrated under reduced pressure and was purified by column chromatography over silica gel (100-200 mesh) to afford the pure product of $\mathbf{2} \mathbf{i}$ in $85 \%$ (214 $\mathrm{mg}$ ) yield. The same procedure was applied for the synthesis of compounds 2a-i, 2n and 20.

$2.2 \mathrm{~b}$ Representative procedure for the synthesis of (Z)-3-alkylidene-phthalans $(\mathbf{2} \mathbf{j}-\mathbf{m})$ in benzene- $d_{6}$ : To an NMR tube equipped with a screw cap containing benzene- $d_{6}(0.50 \mathrm{~mL})$ under argon, 2-(2-(2methoxyphenyl)ethynyl)phenylmethanol (1m, $50.0 \mathrm{mg}$, $0.21 \mathrm{mmol})$ and $\mathrm{Cu}(\mathrm{OTf})_{2} \quad(3.79 \mathrm{mg}, 0.0105 \mathrm{mmol})$ were added. The resulting mixture was heated at $65^{\circ} \mathrm{C}$ and monitored by NMR until the starting material had been consumed. At completion of the reaction, this reaction mixture was filtered through a small plug of silica gel to remove the catalyst. The crude product was purified by silica gel column chromatography (petroleum ether/AcOEt 9.5:0.5) to afford the pure product of $(Z)$-1-(2-methoxy-benzylidene)-1,3dihydro-isobenzofuran $2 \mathrm{~m}$ in $87 \%(43 \mathrm{mg}$ ) yield. The same procedure was applied for the synthesis of compounds $\mathbf{2} \mathbf{j}-\mathbf{m}$.

2.2c (Z)-1-Benzylidene-1,3-dihydro-isobenzofuran (2a): Colourless solid; Mp 120-122 ${ }^{\circ}$; IR (KBr): 3040, 
1612, 1456, 1273, 1064, 817, 750, $685 \mathrm{~cm}^{-1} .{ }^{1} \mathrm{H} \mathrm{NMR}$ $\left(500 \mathrm{MHz}, \mathrm{CDCl}_{3}\right): \delta_{\mathrm{H}} 5.24(\mathrm{~s}, 2 \mathrm{H}) ; 6.49(\mathrm{~s}, 1 \mathrm{H}) ; 7.08-$ $7.12(\mathrm{~m}, 2 \mathrm{H}) ; 7.19(\mathrm{t}, 1 \mathrm{H}, J=7.6 \mathrm{~Hz}) ; 7.27(\mathrm{t}, 1 \mathrm{H}$, $J=6.9 \mathrm{~Hz}) ; 7.34-7.37(\mathrm{~m}, 1 \mathrm{H}) ; 7.39-7.42(\mathrm{~m}, 2 \mathrm{H})$; 7.75-7.77 (m, 2H). ${ }^{13} \mathrm{C}$ NMR $\left(125 \mathrm{MHz}, \mathrm{CDCl}_{3}\right): \delta_{\mathrm{C}}$ 69.1, 101.3, 123.5, 123.9, 125.1, 126.5, 128.1, 128.3, 128.4, 128.9, 132.0, 134.3. 154.1. MS (ESI): $\mathrm{m} / z=$ $209[\mathrm{M}+\mathrm{H}]^{+}$. Anal. Calcd. for $\mathrm{C}_{15} \mathrm{H}_{12} \mathrm{O}: \mathrm{C}, 86.51 ; \mathrm{H}$, 5.81\%. Found: C, 86.75; H, 5.75\%.

2.2d (Z)-1-(4-Methoxy-benzylidene)-1,3-dihydroisobenzofuran (2b): Colourless solid; Mp $122-124^{\circ} \mathrm{C}$; IR (KBr): 3033, 2843, 2331, 1678, 1616, 1550, 1446, 1384, 1271, 1062, 906, 811, 755, $679 \mathrm{~cm}^{-1} .{ }^{1} \mathrm{H} \mathrm{NMR}$ $\left(500 \mathrm{MHz}, \mathrm{CDCl}_{3}\right): \delta_{\mathrm{H}} 3.83(\mathrm{~s}, 3 \mathrm{H}) ; 5.20(\mathrm{~s}, 2 \mathrm{H}) ; 6.35$ $(\mathrm{s}, 1 \mathrm{H}) ; 6.90(\mathrm{~d}, 2 \mathrm{H}, J=9.1 \mathrm{~Hz}) ; 7.05(\mathrm{t}, 2 \mathrm{H}, J=$ $7.6 \mathrm{~Hz}) ; 7.14(\mathrm{t}, 1 \mathrm{H}, J=7.6 \mathrm{~Hz}) ; 7.24(\mathrm{t}, 1 \mathrm{H}, J=$ $7.6 \mathrm{~Hz}) ; 7.67(\mathrm{~d}, 2 \mathrm{H}, J=8.4 \mathrm{~Hz}) .{ }^{13} \mathrm{C}$ NMR $(125 \mathrm{MHz}$, $\left.\mathrm{CDCl}_{3}\right): \delta_{\mathrm{C}} 55.4,69.1,99.6,113.8,123.2,123.8,126.1$, 126.7, 126.9, 127.8, 128.2, 132.3, 154.1, 160.3. MS (ESI): $m / z=239[\mathrm{M}+\mathrm{H}]^{+}$. Anal. Calcd. for $\mathrm{C}_{16} \mathrm{H}_{14} \mathrm{O}_{2}$ : C, 80.65; H, 5.92\%. Found: C, 80.88; H, 5.85\%.

2.2e (Z)-1-(3-Methyl-benzylidene)-1,3-dihydroisobenzofuran (2c): Pink solid; Mp $112-114^{\circ} \mathrm{C}$; IR (KBr): 3042, 2840, 1946, 1698, 1602, 1448, 1383, 1283, 1175, 1069, 881, 782, $691 \mathrm{~cm}^{-1}$. ${ }^{1} \mathrm{H}$ NMR $\left(500 \mathrm{MHz}, \mathrm{CDCl}_{3}\right): \delta_{\mathrm{H}} 2.42(\mathrm{~s}, 3 \mathrm{H}) ; 5.24(\mathrm{~s}, 2 \mathrm{H}) ; 6.48$ (s, 1H); 7.08-7.12 (m, 2H); 7.17-7.19 (m, 2H); 7.27$7.32(\mathrm{~m}, 2 \mathrm{H}) ; 7.56-7.59$ (m, 2H). ${ }^{13} \mathrm{C}$ NMR $(125 \mathrm{MHz}$, $\left.\mathrm{CDCl}_{3}\right): \delta_{\mathrm{C}} 21.6,69.1,101.2,122.3,123.5,123.8$, $125.8,126.5,128.1,128.3$ (2C), 129.7, 132.1, 134.3, 138.0, 154.3. MS (ESI): $m / z=223[\mathrm{M}+\mathrm{H}]^{+}$. Anal. Calcd. for $\mathrm{C}_{16} \mathrm{H}_{14} \mathrm{O}: \mathrm{C}, 86.45 ; \mathrm{H}, 6.35 \%$. Found: C, 86.60; H, 6.30\%.

$2.2 \mathrm{f}$ (Z)-1-(4-Methyl-benzylidene)-1,3-dihydroisobenzofuran (2d): Colourless solid; Mp $96-98^{\circ} \mathrm{C}$; IR (KBr): 2846, 1910, 1605, 1448, 1270, 1193, 1061, 931, $802 \mathrm{~cm}^{-1} .{ }^{1} \mathrm{H}$ NMR $\left(500 \mathrm{MHz}, \mathrm{CDCl}_{3}\right): \delta_{\mathrm{H}} 2.39$ $(\mathrm{s}, 3 \mathrm{H}) ; 5.22(\mathrm{~s}, 2 \mathrm{H}) ; 6.43(\mathrm{~s}, 1 \mathrm{H}) ; 7.08(\mathrm{t}, 2 \mathrm{H}, J=$ $8.4 \mathrm{~Hz}) ; 7.17(\mathrm{t}, 1 \mathrm{H}, J=7.6 \mathrm{~Hz}) ; 7.20(\mathrm{~d}, 2 \mathrm{H}$, $J=7.6 \mathrm{~Hz}) ; 7.24(\mathrm{t}, 1 \mathrm{H}, J=8.4 \mathrm{~Hz}) 7.64(\mathrm{~d}, 2 \mathrm{H}$, $J=8.4 \mathrm{~Hz}) \cdot{ }^{13} \mathrm{C} \mathrm{NMR}\left(125 \mathrm{MHz}, \mathrm{CDCl}_{3}\right): \delta_{\mathrm{C}} 21.4$, $69.0,100.5,123.4,123.8,125.1,126.3,128.0,128.2$, 129.1, 131.5, 132.2, 139.0. 154.3. MS (ESI): $\mathrm{m} / \mathrm{z}=$ $223[\mathrm{M}+\mathrm{H}]^{+}$. Anal. Calcd. for $\mathrm{C}_{16} \mathrm{H}_{14} \mathrm{O}: \mathrm{C}, 86.45 ; \mathrm{H}$, $6.35 \%$. Found: C, $86.25 ; \mathrm{H}, 6.40 \%$.

2.2g (Z)-1-Benzylidene-3-butyl-1,3-dihydroisobenzofuran (2e): Yellow oil; IR (neat) 2955, 2929, 2869, 1655,
$1464,1365,1307,1049,809,761,693,517 \mathrm{~cm}^{-1} \cdot{ }^{1} \mathrm{H}$ NMR $\left(500 \mathrm{MHz}, \mathrm{CDCl}_{3}\right): \delta_{\mathrm{H}} 0.91(\mathrm{t}, 3 \mathrm{H}, J=7.3 \mathrm{~Hz})$; $1.29-1.58(\mathrm{~m}, 4 \mathrm{H}) ; 1.66-1.81(\mathrm{~m}, 4 \mathrm{H}), 1.90-2.01(\mathrm{~m}$, $1 \mathrm{H}) ; 5.60(\mathrm{dd}, 1 \mathrm{H}, J=7.8,3.8 \mathrm{~Hz}) ; 5.88(\mathrm{~s}, 1 \mathrm{H})$; 7.10-7.18 (m, 1H); 7.19-7.23 (m, 1H); 7.24-7.37 $(\mathrm{m}, 1 \mathrm{H}) ; 7.46-7.52(\mathrm{~m}, 1 \mathrm{H}) ; 7.72-7.80(\mathrm{~m}, 2 \mathrm{H}) .{ }^{13} \mathrm{C}$ NMR $\left(125 \mathrm{MHz}, \mathrm{CDCl}_{3}\right): \delta_{\mathrm{C}} 14.0,22.6,27.1,35.7$, 86.1, 95.7, 119.9, 121.2, 125.1, 127.7, 128.1, 128.3, 128.6, 135.0, 136.7, 142.8, 155.8. MS (ESI): $\mathrm{m} / z=$ $265[\mathrm{M}+\mathrm{H}]^{+}$. Anal. Calcd. for $\mathrm{C}_{19} \mathrm{H}_{20} \mathrm{O}: \mathrm{C}, 86.32 ; \mathrm{H}$, 7.63\%. Found: C, 86.15; H, 7.70\%.

2.2h (Z)-1,1-Diethyl-3-pentylidcene-1,3-dihydroisobenzofuran (2f): Yellow oil; IR (neat): 2964, 2930, 2874, 2853, 1679, 1463, 1354, 1296, 1050, $752 \mathrm{~cm}^{-1} \cdot{ }^{1} \mathrm{H}$ NMR (500 MHz, Benzene- $\left.d_{6}\right): \delta_{\mathrm{H}} 0.75(\mathrm{t}, J=7.3 \mathrm{~Hz})$; 0.95 (t, 3H, $J=7.3 \mathrm{~Hz}) ; 1.36-1.76(\mathrm{~m}, 6 \mathrm{H}) ; 1.80-1.95$ $(\mathrm{m}, 2 \mathrm{H}) ; 2.54$ (q, 3H. $J=7.2 \mathrm{~Hz}) 4.94(\mathrm{t}, 1 \mathrm{H}, J=$ 7.2 Hz); 6.71-6.77 (m, 1H); 7.01-7.06 (m, 2H); 7.22$7.28(\mathrm{~m}, 1 \mathrm{H})$. MS (ESI): $m / z=245[\mathrm{M}+\mathrm{H}]^{+}$. Anal. Calcd. for $\mathrm{C}_{17} \mathrm{H}_{24} \mathrm{O}: \mathrm{C}, 83.55 ; \mathrm{H}, 9.90 \%$. Found: $\mathrm{C}$, 83.35; H, $9.95 \%$.

$2.2 \mathrm{i}$ (Z)-3-Benzylidene-1,1-diethyl-1,3-dihydroisobenzofuran (2g): Colourless solid; Mp $62-64^{\circ} \mathrm{C}$; IR (KBr): 2960, 1649, 1462, 1358, 1096, 1051, 939, 820, $761 \mathrm{~cm}^{-1}$. ${ }^{1} \mathrm{H}$ NMR $\left(500 \mathrm{MHz}\right.$, Benzene- $\left.d_{6}\right): \delta_{\mathrm{H}} 0.68$ $(\mathrm{t}, 6 \mathrm{H}, J=7.3 \mathrm{~Hz}) ; 1.53-1.66(\mathrm{~m}, 2 \mathrm{H}) ; 1.80-1.94(\mathrm{~m}$, $2 \mathrm{H}) ; 5.95(\mathrm{~s}, 1 \mathrm{H}) ; 6.66-6.74(\mathrm{~m}, 1 \mathrm{H}) ; 7.00-7.14(\mathrm{~m}$, $3 \mathrm{H}) ; 7.20-7.26(\mathrm{~m}, 1 \mathrm{H}) ; 7.30-7.40(\mathrm{~m}, 2 \mathrm{H}) ; 8.00-8.05$ $(\mathrm{m}, 2 \mathrm{H}) .{ }^{13} \mathrm{C}$ NMR $\left(125 \mathrm{MHz}\right.$, Benzene- $\left.d_{6}\right): \delta_{\mathrm{C}} 7.9$, 33.0, 94.7, 96.0, 120.1, 121.1, 125.4, 128.0, 128.4, 128.6, 136.5, 137.6, 144.5, 156.0. MS (ESI): $m / z=$ $265[\mathrm{M}+\mathrm{H}]^{+}$. Anal. Calcd. for $\mathrm{C}_{19} \mathrm{H}_{20} \mathrm{O}: \mathrm{C}, 86.32 ; \mathrm{H}$, 7.63\%. Found: C, 86.50; H, 7.55\%.

2.2j (Z)-1-(3H-Isobenzofuran-1-ylidene)-propan-2-one (2h): Yellow oil; IR (neat): 1671, 1624, 1590, $706 \mathrm{~cm}^{-1} .{ }^{1} \mathrm{H}$ NMR $\left(500 \mathrm{MHz}, \mathrm{CDCl}_{3}\right): \delta_{\mathrm{H}} 2.46(\mathrm{~s}$, $3 \mathrm{H}) ; 5.58(\mathrm{~s}, 2 \mathrm{H}) ; 5.83(\mathrm{~s}, 1 \mathrm{H}) ; 7.35-7.71(\mathrm{~m}, 4 \mathrm{H}) .{ }^{13} \mathrm{C}$ NMR $\left(125 \mathrm{MHz}, \mathrm{CDCl}_{3}\right): \delta_{\mathrm{C}} 31.4,77.0,98.2,121.8$, 129.2, 132.0, 135.6, 141.8, 167.4, 197.6. MS (ESI): $m / z=175[\mathrm{M}+\mathrm{H}]^{+}$. Anal. Calcd. for $\mathrm{C}_{11} \mathrm{H}_{10} \mathrm{O}_{2}: \mathrm{C}$, 75.84; H, 5.79\%. Found: C, 76.05; H, 5.71\%.

2.2k (Z)-5-Benzylidene-5,7-dihydro-furo [3',4':4,5]benzo [1,2-d][1,3]dioxole (2i): Pale yellow solid; Mp 132$134^{\circ} \mathrm{C}$; IR (KBr): 2898, 1733, 1632, 1481, 1367, 1291, 1152, 1035, 860, $757 \mathrm{~cm}^{-1} .{ }^{1} \mathrm{H}$ NMR $(500 \mathrm{MHz}$, $\left.\mathrm{CDCl}_{3}\right): \delta_{\mathrm{H}} 5.11(\mathrm{~s}, 2 \mathrm{H}) ; 5.93(\mathrm{~s}, 2 \mathrm{H}) ; 6.36(\mathrm{~s}, 1 \mathrm{H}) ; 6.60$ $(\mathrm{d}, 2 \mathrm{H}, J=10.7 \mathrm{~Hz}) ; 7.32-7.38(\mathrm{~m}, 3 \mathrm{H}) ; 7.69(\mathrm{~d}, 2 \mathrm{H}$, 
$J=6.8 \mathrm{~Hz}) \cdot{ }^{13} \mathrm{C} \mathrm{NMR}\left(125 \mathrm{MHz}, \mathrm{CDCl}_{3}\right): \delta_{\mathrm{C}} 69.0$, 101.0, 101.4, 104.6, 105.2, 121.5, 124.8, 126.3, 128.4, 128.7, 134.2, 146.1, 147.5, 152.7. MS (ESI): $m / z=$ $253[\mathrm{M}+\mathrm{H}]^{+}$. Anal. Calcd. for $\mathrm{C}_{16} \mathrm{H}_{12} \mathrm{O}_{3}: \mathrm{C}, 76.18 ; \mathrm{H}$, 4.79\%. Found: C, 75.99; H, 4.85\%.

2.21 1-Methylene-1,3-dihydroisobenzofuran $(\mathbf{2} \mathbf{j}):{ }^{1} \mathrm{H}$ NMR $\left(500 \mathrm{MHz}\right.$, Benzene- $\left.d_{6}\right): \delta_{\mathrm{H}} 4.54(\mathrm{~s}, 1 \mathrm{H}) ; 4.71(\mathrm{~s}$, $1 \mathrm{H})$; $4.75(\mathrm{~s}, 2 \mathrm{H})$; 6.62-6.65 (m, 1H); 6.91-6.96 (m, $2 \mathrm{H})$; 7.19-7.21 (m, 1H). ${ }^{13} \mathrm{C}$ NMR $(125 \mathrm{MHz}$, Benzene$\left.d_{6}\right): \delta_{\mathrm{C}} 73.4,78.0,120.6,121.2,128.2,128.7,134.1$, $140.6,162.5$.

$2.2 \mathrm{~m}$ 1-Methyl-3-methylene-1,3-dihydroisobenzofuran (2k): ${ }^{1} \mathrm{H}$ NMR $\left(500 \mathrm{MHz}\right.$, Benzene- $\left.d_{6}\right): \delta_{\mathrm{H}} 1.18$ (d, $3 \mathrm{H}, J=6.8 \mathrm{~Hz}$ ); $4.55(\mathrm{~s}, 1 \mathrm{H}) ; 4.72(\mathrm{~s}, 1 \mathrm{H}) ; 5.17$ (q, $1 \mathrm{H}, J=5.9 \mathrm{~Hz}) ; 6.69(\mathrm{t}, 1 \mathrm{H}, J=4.4 \mathrm{~Hz}) ; 6.96-7.00$ $(\mathrm{m}, 2 \mathrm{H}) ; 7.20(\mathrm{t}, 1 \mathrm{H}, J=4.4 \mathrm{~Hz}) .{ }^{13} \mathrm{C}$ NMR $(125 \mathrm{MHz}$, Benzene- $\left.d_{6}\right): \delta_{\mathrm{C}} 21.2,77.9,80.7,120.7,121.0,128.2$, 128.8, 134.0, 145.0, 161.3.

2.2n (3-Methylene-1,3-dihydroisobenzofuran-1-yl) methanamine (2I): ${ }^{1} \mathrm{H}$ NMR $(500 \mathrm{MHz}$, Benzene$\left.d_{6}\right): \delta_{\mathrm{H}} 0.60$ (brs, $\left.2 \mathrm{H}\right) ; 2.59-2.64(\mathrm{~m}, 1 \mathrm{H}) ; 2.74-2.77$ $(\mathrm{m}, 1 \mathrm{H}) ; 4.54(\mathrm{~s}, 1 \mathrm{H}) ; 4.70(\mathrm{~s}, 1 \mathrm{H}) ; 5.04(\mathrm{t}, 1 \mathrm{H}, J=$ $4.4 \mathrm{~Hz}) ; 6.75(\mathrm{t}, 1 \mathrm{H}, J=4.9 \mathrm{~Hz}) ; 6.95(\mathrm{t}, 2 \mathrm{H}, J=$ $3.9 \mathrm{~Hz}) ; 7.21(\mathrm{t}, 1 \mathrm{H}, J=5.9 \mathrm{~Hz}) .{ }^{13} \mathrm{C} \mathrm{NMR}(125 \mathrm{MHz}$, Benzene- $\left.d_{6}\right): \delta_{\mathrm{C}} 46.9,18.0,86.3,120.7,121.5$, 128.2, 128.8, 135.0, 141.7, 161.7.

2.20 (Z)-1-(2-Methoxy-benzylidene)-1,3-dihydroisobenzofuran (2m): Brown paste; IR (neat): 3308, 2938, 2189, 1635, 1427, 1327, 1200, 1076, $878 \mathrm{~cm}^{-1}$. ${ }^{1} \mathrm{H}$ NMR $\left(500 \mathrm{MHz}\right.$, Benzene- $\left.d_{6}\right): \delta_{\mathrm{H}} 3.25(\mathrm{~s}, 3 \mathrm{H})$; 4.92 (s, 2H); 6.48-7.05 (m, 8H); 7.92-7.93 (m, 1H). ${ }^{13} \mathrm{C}$ NMR $\left(125 \mathrm{MHz}\right.$, Benzene- $\left.d_{6}\right): 54.8,68.5,107.0$, $111.2,120.4,123.7,123.8,126.2,128.3,128.5,128.8$, 129.4, 129.6, 132.6, 151.4, 157.6. MS (ESI): $\mathrm{m} / z=$ $239[\mathrm{M}+\mathrm{H}]^{+}$. Anal. Calcd. for $\mathrm{C}_{16} \mathrm{H}_{14} \mathrm{O}_{2}: \mathrm{C}, 80.65 ; \mathrm{H}$, $5.92 \%$. Found: C, 80.85 ; H, 5.86\%.

$2.2 \mathrm{p}$ (Z)-2-(3H-Isobenzofuran-1ylidenemethyl)-pyridine (2n): Yellow oil; IR (neat): 3401, 3358, 1612, 1430, 1300, 1211, 1076, $691 \mathrm{~cm}^{-1}$. ${ }^{1} \mathrm{H}$ NMR $(500 \mathrm{MHz}$, $\left.\mathrm{CDCl}_{3}\right): \delta_{\mathrm{H}} 5.52(\mathrm{~s}, 2 \mathrm{H}) ; 6.22(\mathrm{~s}, 1 \mathrm{H}) ; 7.00(\mathrm{dd}, 1 \mathrm{H}$, $J=8.1 \mathrm{~Hz}, J=1.0 \mathrm{~Hz}) ; 7.31-7.38(\mathrm{~m}, 3 \mathrm{H}) ; 7.60$ $7.65(\mathrm{~m}, 2 \mathrm{H}) ; 8.06(\mathrm{~d}, 1 \mathrm{H}, J=8.0 \mathrm{~Hz}) 8.50(\mathrm{~d}, 1 \mathrm{H}$, $J=4.5 \mathrm{~Hz}) .{ }^{13} \mathrm{C}$ NMR $\left(125 \mathrm{MHz}, \mathrm{CDCl}_{3}\right): \delta_{\mathrm{C}} 76.8$, 89.4, 121.0, 122.7, 126.6, 127.1, 127.6, 128.2, 137.0, 143.4, 148.9, 152.7, 154.9, 159.9. MS (ESI): $\mathrm{m} / z=$
$210[\mathrm{M}+\mathrm{H}]^{+}$. Anal. Calcd. for $\mathrm{C}_{14} \mathrm{H}_{11} \mathrm{ON}: \mathrm{C}, 80.36 ; \mathrm{H}$, 5.30; N, 6.69\%. Found: C, 80.55; H, 5.27; N, 6.79\%.

2.2q (Z)-2-(3H-Isobenzofuran-1ylidenemethyl)-pyrazine (2o): Yellow oil; IR (neat): 3411, 3351, 1602, 1420, 1310, 1210, 1077, $699 \mathrm{~cm}^{-1}$. ${ }^{1} \mathrm{H}$ NMR $(500 \mathrm{MHz}$, $\left.\mathrm{CDCl}_{3}\right): \delta_{\mathrm{H}} 5.60(\mathrm{~s}, 2 \mathrm{H}) ; 6.15(\mathrm{~s}, 1 \mathrm{H}) ; 7.36-7.50(\mathrm{~m}$, $3 \mathrm{H}) ; 7.65(\mathrm{dd}, 1 \mathrm{H}, J=8.0,1.0 \mathrm{~Hz}) ; 8.25(\mathrm{~s}, 1 \mathrm{H}) ; 8.47$ (s, $1 \mathrm{H}) ; 9.31(\mathrm{~s}, 1 \mathrm{H}),{ }^{13} \mathrm{C}$ NMR $\left(125 \mathrm{MHz}, \mathrm{CDCl}_{3}\right): \delta_{\mathrm{C}}$ 76.5, 85.9, 119.7, 126.6, 127.1, 127.6, 128.2, 143.4, 152.7, 157.6, 160.1, 166.9. MS (ESI): $m / z=211$ $[\mathrm{M}+\mathrm{H}]^{+}$. Anal. Calcd. for $\mathrm{C}_{13} \mathrm{H}_{10} \mathrm{ON}_{2}: \mathrm{C}, 74.27 ; \mathrm{H}$, 4.79; N, 13.32\%. Found: C, 74.55; H, 4.72; N, $13.22 \%$.

\subsection{Animals and drug dosage}

2.3a Animals: Inbred albino mice (Swiss strain) of adult gender weighing 20-25 g were used for the study. The mice were kept in clean polypropylene cages with free access to standard pellet diet and water (ab libitum), under standardized housing conditions (natural light-dark cycle, temperature $23 \pm 1^{\circ} \mathrm{C}$, relative humidity $55 \pm 5 \%$ ). After 7 days of adaptation to laboratory conditions, the animals were randomly assigned to 12 experimental groups of 5 mice each. Each mouse was used only once. All tests were performed between 08:00 and 16:00 h. All efforts were made to minimize animal suffering and to use only the number of animals necessary to produce reliable scientific data. The experimental protocols and procedures listed below conformed to the Guide for the Care and Use of Laboratory Animals and approved by the Institutional Ethics Committee. Mice equivalent doses in $\mathrm{mg} / \mathrm{kg}$ body weight of clinical doses were calculated as $\mathrm{mg} / \mathrm{kg}$ body weight with the help of standard tables. ${ }^{10}$

2.3b Chemical and administration of compounds: The synthesized compounds $(10 \mathrm{mg} / \mathrm{kg})$, and citalopram as a reference antidepressant drug $(10 \mathrm{mg} / \mathrm{kg})$ were dissolved in DMSO. The drugs were injected intraperitoneally (ip) in a standard volume of $0.5 \mathrm{~mL} / 20 \mathrm{~g}$ body weight, $1 \mathrm{~h}$ prior to the test.

\subsection{Evaluation of antidepressant activity}

2.4a Porsolt's behavioural despair test (Forced swim test): The method described by Porsolt et al. was used in our study. ${ }^{11}$ Each animal was placed individually in a $5 \mathrm{~L}$ glass beakers, filled with water up to a height of $15 \mathrm{~cm}$ and were observed for duration of $6 \mathrm{~min}$. The duration of immobility was recorded during the last 
4 min of the observation period. The mouse was considered immobile when it floated motionlessly or made only those moments necessary to keep its head above the water surface. Water was changed after each test.

2.4b Tail suspension test: Antidepressant-like activity of the test compounds was screened using the tail suspension test similar to that described by Steru et al. ${ }^{12}$ Mice were dangled from their tail using adhesive tape placed approximately $1 \mathrm{~cm}$ from the tip of the tail attached to a applicator stick and hung approximately $30 \mathrm{~cm}$ above a table. Mice were considered immobile only when they fail to make any struggling movements and hung passively. Immobility time for each animal was scored by stopwatch during the last $4 \mathrm{~min}$ of a 6 min test. ${ }^{12 \mathrm{a}}$

\subsection{Statistical analysis}

The obtained data were analysed using one-way analysis of variance (ANOVA) followed by Dunnet's multiple comparison test. The results are presented as mean \pm Standard error of means (SEM). Differences between data sets were considered as significant when $P<0.001$.

\subsection{Molecular docking methodology}

2.6a Preparation of the analogs and receptors: All analogues were drawn as 2D structures with ChemDraw Ultra version 9.0. Before docking with the GLIDE and the GOLD each analog was prepared using LigPrep, an application that is available through Maestro 7.5. After employing the energy minimization through Macro model application, each 3D structure was saved as an SD file (*.spf), and combined into a single analogs library SD file, to be used for docking experiments. 2.6b GLIDE docking: Each homology receptor was prepared by the protein preparation mode of GLIDE and the receptor grid was generated by specifying Asp89 as a central residue and selecting extra precision docking within $20 \AA ̊$ of Asp89. After this step the G-scores were determined and the docking poses of each analogues were visually inspected using GLIDE pose viewer.

2.6c GOLD docking: As input file for the GOLD docking, both homology models were used as a PDB file. In the GOLD Wizard, set-up hydrogen was added and the binding site was defined as the residues that are falling within $15 \AA$ of Asp89. The GOLD score was chosen as fitness function. After docking was finished the top-scoring receptor-analogues complexes were visually inspected.

2.6d Visualization of selected docking posses: All final receptor-analogues complexes, obtained from three different programs were visualized using PyMOL viewer only. Hydrogen bonds, hydrophobic interactions and distance for each hydrogen bond were indicated by dashed lines between the atoms involved, while hydrophobic contacts are represented by an arc with spokes radiating towards the ligand atoms they contact.

\section{Results and discussion}

\subsection{Chemistry}

3.1a Synthesis: As part of our ongoing research in the synthesis of novel heterocycles, ${ }^{13}$ we have previously established, that $5 \mathrm{~mol} \%$ of $\mathrm{Cu}(\mathrm{OTf})_{2}$ in toluene was effective for the 5-exo-dig regioisomeric cycloisomerization of 2-(ethynyl)benzyl alcohols leading to phthalan derivatives. ${ }^{13 \mathrm{~h}}$ Various substituted 2-(ethynyl)benzyl alcohols reacted under this

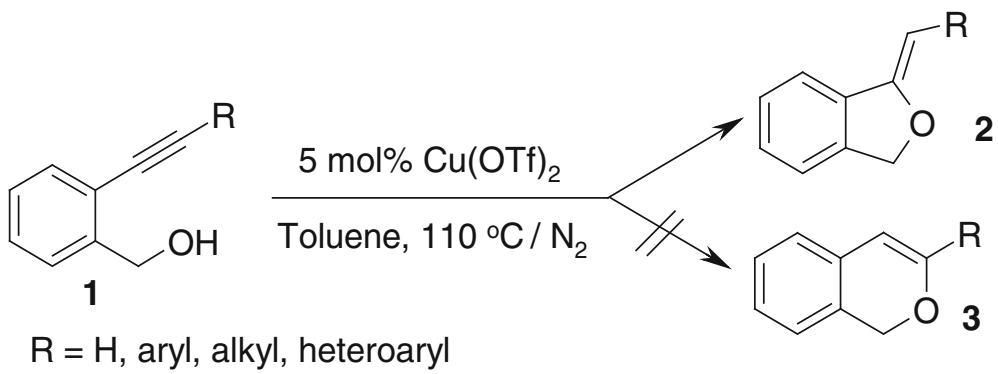

Scheme 1. $\mathrm{Cu}(\mathrm{OTf})_{2}$ catalysed synthesis of isobenzofuran 2 . 
Table 1. Copper(II) catalysed synthesis of phthalans via cycloisomerisation of 2(ethynyl)benzyl alcohols. ${ }^{a}$

\begin{tabular}{|c|c|c|c|c|}
\hline Entry & $\begin{array}{l}\text { 2-(Ethynyl)benzyl } \\
\text { alcohol (1) }\end{array}$ & Phthalan (2) ${ }^{\mathrm{b}}$ & Time (min) & Yield $(\%)^{\mathrm{c}}$ \\
\hline & & & & \\
\hline 1 & & & 20 & 92 \\
\hline
\end{tabular}

$1 \mathbf{a}$

2
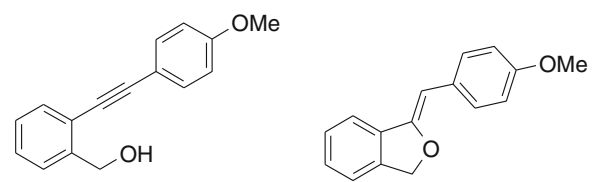

20

95

1b

$2 b$

3
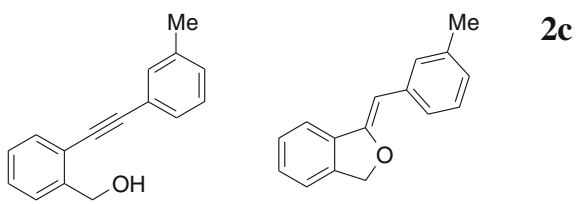

45

94

1c

4
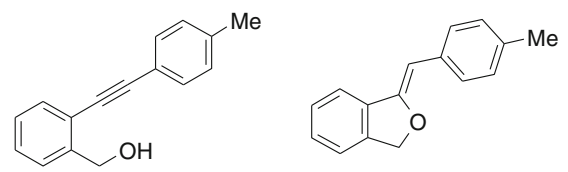

45

95

1d

2d

1e

6<smiles>CCC(O)(CC)c1ccccc1C#CBr</smiles><smiles>CCC1(CC)OC(=CC(C)(C)C)c2ccccc21</smiles>

$2 f$

1f

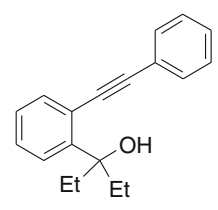<smiles>CCC1(CC)O/C(=C\c2ccccc2)c2ccccc21</smiles>

$2 \mathrm{~g}$

$1 g$<smiles>CC(=O)C#Cc1ccccc1CO</smiles><smiles>CC(=O)C=C1OCc2ccccc21</smiles>

$2 \mathrm{~h}$ 
Table 1. (continued)

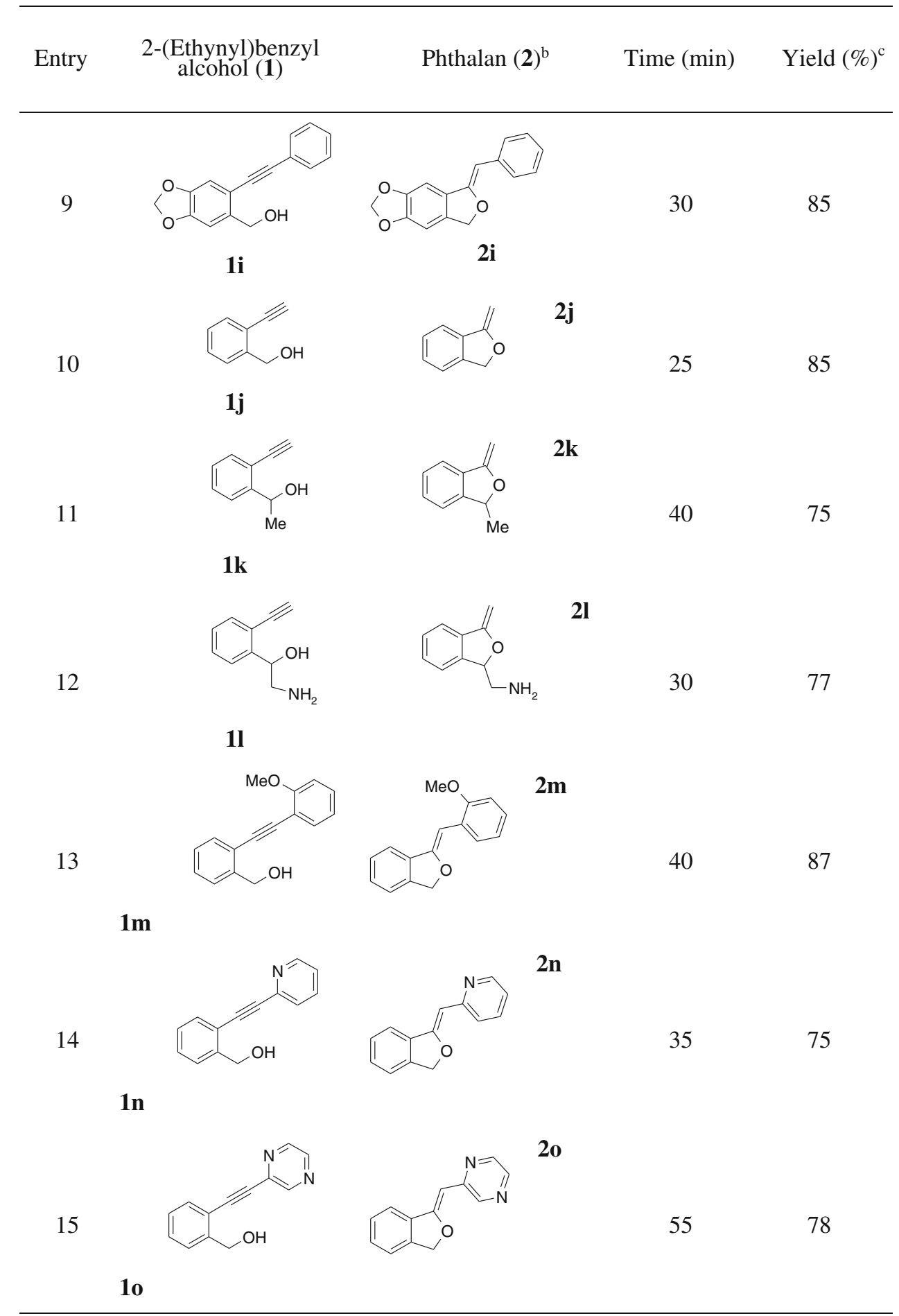

${ }^{\mathrm{a}} \mathrm{All}$ reactions were carried out at $110^{\circ} \mathrm{C}$ in toluene using $5 \mathrm{~mol} \%$ of $\mathrm{Cu}(\mathrm{OTf})_{2}$ under nitrogen atmosphere.

${ }^{\mathrm{b}}$ All products were characterized by IR, ${ }^{1} \mathrm{H}$ NMR, ${ }^{13} \mathrm{C}$ NMR and mass spectroscopy.

${ }^{\mathrm{c}}$ Isolated yield.

${ }^{\mathrm{d}}$ The reaction was carried out in benzene- $d_{6}$ solvent at $65^{\circ} \mathrm{C}$

condition and afforded the corresponding phthalan derivatives (2a-2o) in high yield (scheme 1, table 1).
The results revealed that alkynes possessing electronrich groups enhance the cyclization and generally higher yields are obtained at a shorter reaction time 


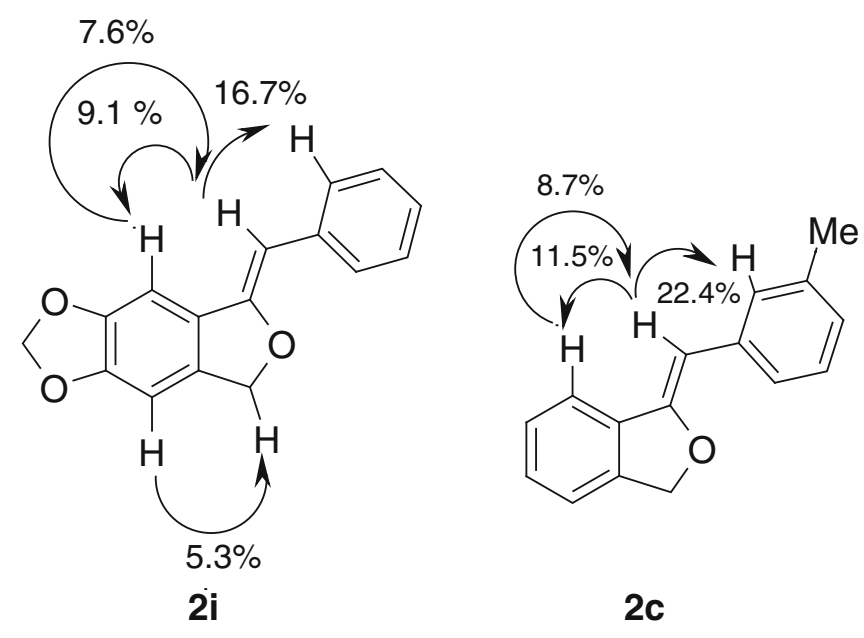

Figure 2. 1-D nOe enhancement of compounds $\mathbf{2} \mathbf{i}$ and $\mathbf{2 c}$.

(entries 2-4). The presence of substituent at the benzylic position did have deleterious effects on the cyclization; primary alcohols underwent cyclization significantly (entries 1-4, 8-10 and 13-15) in terms of shorter reaction time and higher yields, than secondary alcohols (entries 5, 11 and 12), which in turn react more efficiently than their tertiary counterparts (entries 6 and 7). The protocol was found to be chemoselective. For example, when substrate $\mathbf{1 l}$ was allowed to react with $\mathrm{Cu}(\mathrm{OTf})_{2}$, it was found that only the hydroxyl group underwent cycloisomerization to afford the product $2 \mathbf{l}$ leaving the amine group intact. A more complex heteroaromatic synthesis was also achieved under our reaction condition. For example, the piperanol derived substrate $1 \mathbf{i}$ gave excellent yield of the product $2 \mathbf{i}$ under the same reaction conditions. Substrates possessing heteroaromatic motif like pyridine $\mathbf{1 n}$ and pyrazine 1o were well tolerated under our reaction conditions (entries 14 and 15). A preparative scale-catalytic reaction was performed for the reaction of $\mathbf{2} \mathbf{j}-\mathbf{2 m}$ in benzene- $d_{6}$, since these compounds readily underwent decomposition in $\mathrm{CDCl}_{3}$ solvent.

3.1b Structural and stereochemical assignment of (Z)phthalan derivatives: The Z-configuration of phthalan was assigned from comparison of the chemical shifts of the vinylic protons with those reported for sim- ilar compounds. ${ }^{14}$ The $(Z)$-stereochemistry of phthalan $2 \mathbf{i}$ has been assigned using a 1D-nOe experiment. Selective irradiation of the vinylic proton $\left(\delta_{\mathrm{H}}=6.36 \mathrm{ppm}\right)$ effected the enhancement of the signals of $\mathrm{C}_{4}-\mathrm{H}\left(\delta_{\mathrm{H}}=\right.$ $6.62 \mathrm{ppm})$ by $9.1 \%$ and ortho-phenyl proton $\left(\delta_{\mathrm{H}}=\right.$ $7.69 \mathrm{ppm})$ by $16.7 \%$ respectively. Irradiation of $\mathrm{C}_{4}-\mathrm{H}$ effected the enhancement of vinylic proton $(7.5 \%)$ and no enhancement of ortho-phenyl proton. This observation confirmed that the vinylic proton is cis to $\mathrm{C}_{4}$ $\mathrm{H}$, thus the compound $\mathbf{2 i}$ is $Z$-configured. Similarly, for compound $2 \mathrm{c}$, irradiation of vinylic proton $\left(\delta_{\mathrm{H}}\right.$ $=6.48 \mathrm{ppm}$ ) effected the enhancement of both $\mathrm{C}_{4}-\mathrm{H}$ $\left(\delta_{\mathrm{H}}=7.12 \mathrm{ppm}\right)$ by $11.5 \%$ and ortho-phenyl proton $\left(\delta_{\mathrm{H}}=7.59 \mathrm{ppm}\right)$ by $22.4 \%$. Irradiation of $\mathrm{C}_{4}-\mathrm{H}$ proton however effected the enhancement of vinylic proton by $8.7 \%$ and no enhancement of the ortho-phenyl proton. These facts confirmed the cis relationship of the vinylic proton and $\mathrm{C}_{4}-\mathrm{H}$, thus favouring the $\mathrm{Z}$-configuration (figure 2). The stereochemistry of other phthalans is assigned by analogy to $\mathbf{2 i}$ and $\mathbf{2 c}$.

In IR spectra, a peak observed at 1620 to $1650 \mathrm{~cm}^{-1}$ for all compounds, revealed the presence of an ether linkage. In ${ }^{1} \mathrm{H}$ NMR spectra, all products exhibited a sharp singlet between $\delta_{\mathrm{H}} 4.75$ (for unsubstituted olefins) and $6.43 \mathrm{ppm}$ (for substituted olefins), indicated the presence of vinylic proton. In ${ }^{13} \mathrm{C}$ NMR spectra, a peak at $\delta \mathrm{c} 120-125 \mathrm{ppm}$ ascertained the presence of olefinic carbon, characteristic of the exocyclic alkylidene carbon of isobenzofuran (see supporting information). All these findings, confirmed the formation of phthalans.

3.1c Mechanism: The reason for the exclusive formation of the five-member ring over their six-member counterpart was not clear. However, a tentative mechanism, on the basis of the obtained results is proposed (scheme 2), according to which a six-member transition state $\mathbf{4}$ was formed via a bidentate complexation of [Cu] with the hydroxyl group and $\alpha$-carbon of the alkyne of 1 . As a consequence, a partial depletion of electron density at the $\beta$-carbon of the acetylene function drives the nucleophilic attack of the pendant hydroxyl group towards the $\beta$-carbon leading to the five-member intermediate 5. Subsequent protodecupration of $\mathbf{5}$ results in the formation of phthalan $\mathbf{2}$.

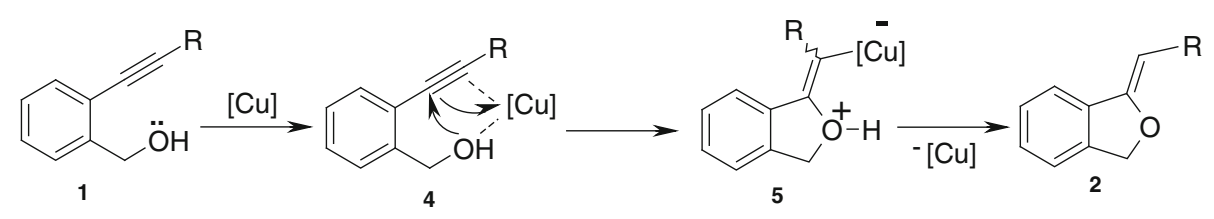

Scheme 2. Plausible mechanism for the formation of isobenzofuran 2. 
Table 2. Antidepressant activity of compounds (2a-2o) by forced swim test.

\begin{tabular}{lccc}
\hline S.No & Compound & Duration of immobility (s) & \% change from control \\
\hline 1 & $\mathbf{2 a}$ & $086.0 \pm 1.41 * *$ & -57.0 \\
2 & $\mathbf{2 b}$ & $154.0 \pm 1.41^{* *}$ & -23.0 \\
3 & $\mathbf{2 c}$ & $135.0 \pm 5.09 * *$ & -32.5 \\
4 & $\mathbf{2 d}$ & $104.0 \pm 1.07 * *$ & -48.0 \\
5 & $\mathbf{2}$ & $85.0 \pm 1.41^{* *}$ & -57.5 \\
6 & $\mathbf{2 f}$ & $035.0 \pm 1.41^{* *}$ & -82.5 \\
7 & $\mathbf{2 g}$ & $072.0 \pm 0.71 * *$ & -64.0 \\
8 & $\mathbf{2 h}$ & $093.6 \pm 1.07 * *$ & -53.2 \\
9 & $\mathbf{2 i}$ & $102.8 \pm 1.71 * *$ & -49.0 \\
10 & $\mathbf{2 j}$ & $195.8 \pm 1.28 * *$ & -02.1 \\
11 & $\mathbf{2 k}$ & $180.0 \pm 1.41 * *$ & -10.0 \\
12 & $\mathbf{2}$ & $145.0 \pm 1.14 * *$ & -27.5 \\
13 & $\mathbf{2 m}$ & $151.0 \pm 1.00^{* *}$ & -63.5 \\
14 & $\mathbf{2 n}$ & $073.0 \pm 0.71 * *$ & -64.5 \\
15 & $\mathbf{2 0}$ & $071.0 \pm 0.71 * *$ & -71.5 \\
16 & Citalopram (10 mg/kg) & $057.0 \pm 0.73 * *$ & - \\
17 & Control (Vehicle) & $200.0 \pm 6.88^{* *}$ & \\
\hline
\end{tabular}

Data were analysed by one way ANNOVA followed by Dunnet's t test.

P values: $* *<0.001$

**are more significant

\section{Pharmacology}

\subsection{Evaluation of antidepressant activity}

All the synthesized compounds (2a-2o) were evaluated for their antidepressant activity by forced swim and tail suspension tests. ${ }^{11,12}$ The results were shown in tables 2 and 3 for forced swim test and tail suspension test, respectively.

As shown in table 2, the reference drug citalopram $(10 \mathrm{mg} / \mathrm{kg})$ and the test compounds $(10 \mathrm{mg} / \mathrm{kg})$ significantly shortened the immobility time of mice in forced swim test. Similarly in tail suspension test, citalopram

Table 3. Antidepressant activity of compounds (2a-2o) by tail suspension test.

\begin{tabular}{lccc}
\hline S.No & Compounds & Duration of immobility (s) & \% change from control \\
\hline 1 & $\mathbf{2 a}$ & $080.0 \pm 0.71^{*}$ & -54.29 \\
2 & $\mathbf{2 b}$ & $125.8 \pm 0.83^{*}$ & -28.11 \\
3 & $\mathbf{2 c}$ & $134.4 \pm 1.20^{*}$ & -23.20 \\
4 & $\mathbf{2 d}$ & $139.6 \pm 1.21^{*}$ & -20.23 \\
5 & $\mathbf{2}$ & $081.6 \pm 1.07^{*}$ & -53.37 \\
6 & $\mathbf{2}$ & $040.0 \pm 1.41^{*}$ & -77.14 \\
7 & $\mathbf{2 g}$ & $070.8 \pm 1.23^{*}$ & -59.54 \\
8 & $\mathbf{2 h}$ & $083.8 \pm 1.28^{*}$ & -52.11 \\
9 & $\mathbf{2}$ & $095.8 \pm 0.87^{*}$ & -45.23 \\
10 & $\mathbf{2}$ & $180.4 \pm 0.92^{*}$ & +03.09 \\
11 & $\mathbf{2}$ & $169.2 \pm 3.78^{* *}$ & -07.31 \\
12 & $\mathbf{2}$ & $139.0 \pm 1.00^{*}$ & -20.57 \\
13 & $\mathbf{2 m}$ & $129.2 \pm 1.65^{*}$ & -26.17 \\
14 & $\mathbf{2 n}$ & $071.0 \pm 0.71^{* *}$ & -59.43 \\
15 & $\mathbf{2 0}$ & $0.78 .2 \pm 0.87^{*}$ & -55.31 \\
16 & Citalopram (10 mg/kg) & $068.0 \pm 1.25^{*}$ & -61.14 \\
17 & Control (Vehicle) & $175.0 \pm 4.79^{*}$ & - \\
\hline
\end{tabular}

Data were analysed by one way ANNOVA followed by Dunnet's t test.

P values: $*<0.5, * *<0.001$.

$* *$ are more significant 
Table 4. Calculated Lipinski's rule of five for the compounds (2a-2o).

\begin{tabular}{lccccc}
\hline S.No & Compounds & M.W & $\log \mathrm{p}^{\mathrm{b}}$ & $\mathrm{n} \mathrm{ON}^{\mathrm{c}}$ & $\mathrm{n} \mathrm{ONH}^{\mathrm{d}}$ \\
\hline 1 & $\mathbf{2 a}$ & 208.26 & 3.755 & 1 & 0 \\
2 & $\mathbf{2 b}$ & 238.29 & 3.812 & 2 & 0 \\
3 & $\mathbf{2 c}$ & 222.29 & 4.179 & 1 & 0 \\
4 & $\mathbf{2 d}$ & 222.29 & 4.203 & 1 & 0 \\
5 & $\mathbf{2}$ & 264.37 & 5.685 & 1 & 0 \\
6 & $\mathbf{2}$ & 244.38 & 5.918 & 1 & 0 \\
7 & $\mathbf{2 g}$ & 264.37 & 5.57 & 1 & 0 \\
8 & $\mathbf{2 h}$ & 174.20 & 1.468 & 2 & 0 \\
9 & $\mathbf{2}$ & 252.27 & 3.621 & 3 & 0 \\
10 & $\mathbf{2 j}$ & 132.16 & 2.048 & 1 & 0 \\
11 & $\mathbf{2}$ & 146.19 & 2.411 & 1 & 0 \\
12 & $\mathbf{2}$ & 161.21 & 0.840 & 2 & 0 \\
13 & $\mathbf{2 m}$ & 238.29 & 3.584 & 2 & 0 \\
14 & $\mathbf{2 n}$ & 209.25 & 2.405 & 2 & 0 \\
15 & $\mathbf{2 0}$ & 210.24 & 2.108 & 3 & 3 \\
16 & Citalopram & 324.39 & 3.17 & 3 & \\
\hline
\end{tabular}

${ }^{a}$ Molecular weight.

${ }^{\mathrm{b}}$ Calculated lipophilicity.

${ }^{\mathrm{c}}$ Number of hydrogen bond acceptor.

${ }^{\mathrm{d} N u m b e r}$ of hydrogen bond donor

and all test compounds shortened the immobility time of mice (table 3 ). The results of the animal study indicated that all the compounds showed significant activity except compound $\mathbf{2} \mathbf{j}$. Compound $\mathbf{2 f}$ showed more potent activity than citalopram.

\subsection{Lipinski rule of five and drug-likeness profile}

All the compounds were submitted for the analysis of Lipinski rule of five that indicates if a compound could be an orally active drug in human. ${ }^{15}$ Our in vivo results showed that all active compounds fulfilled this rule, similar to the clinically used drug, citalopram (molecular weight $=208.26$ to $264.37 \mathrm{~g} / \mathrm{mol}, \log \mathrm{p}=2.05-$ $5.6, \mathrm{nON}=1-3$, and $\mathrm{nOHNH}=0-2$ ) (table 4). However a couple of points need to be noted. Compound
$2 \mathbf{i}$ which is closest to citalopram in $\log \mathrm{p}, \mathrm{nON}, \mathrm{nONH}$ and M.W values is less active. The most active compound of our study is $\mathbf{2} \mathbf{f}$ which has the highest $\log \mathrm{p}$ value in the series. Compounds $\mathbf{2 e}$ and $\mathbf{2 g}$ are slightly less lipophilic and less active. On the other hand, compound $\mathbf{2 h}$ which is much less lipophilic $(\log \mathrm{p}=1.468)$ than $2 \mathrm{e}$ does not lag far behind the latter in activity. There are many approaches that assess a compound's drug-likeness based on topological descriptors, fingerprints of molecular drug-likeness, structural keys or other properties like $\mathrm{c} \log \mathrm{p}$ and molecular weight. ${ }^{16}$ In the present work, we used molinspiration program for calculating the fragment based drug-likeness of all the compounds and compared with citalopram (figure 3). Interestingly in this analysis compounds $\mathbf{2 e}$, $2 f$ and $2 \mathrm{~g}$ with a drug-likeness score of $-0.25,-0.28$

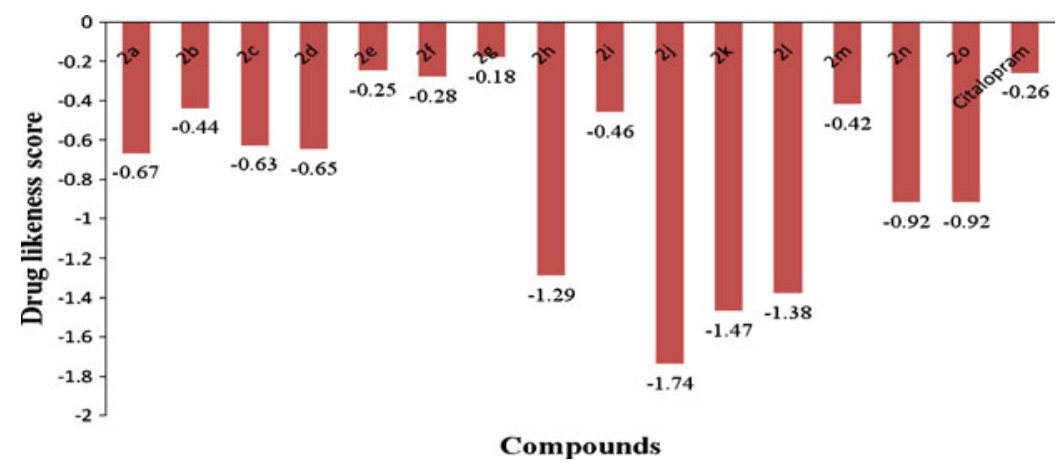

Figure 3. Drug-likeness score of compounds (2a-2o). 
Table 5. Docking study of compounds (2a-2o) on serotonin binding site of arabinase receptor model (PDB code: 2WRZ).

\begin{tabular}{lcccc}
\hline S.No & Compound & Gold score & Glide score & Docking score \\
\hline 1 & $\mathbf{2 a}$ & 38.08 & -38.20 & -6.34 \\
2 & $\mathbf{2 b}$ & 36.51 & -40.96 & -5.96 \\
3 & $\mathbf{2 c}$ & 39.72 & -40.07 & -6.14 \\
4 & $\mathbf{2 d}$ & 34.90 & -38.97 & -6.08 \\
5 & $\mathbf{2 e}$ & 42.51 & -40.87 & -6.05 \\
6 & $\mathbf{2 f}$ & 43.39 & -36.22 & -5.88 \\
7 & $\mathbf{2 g}$ & 43.04 & -43.14 & -6.11 \\
8 & $\mathbf{2 h}$ & 33.96 & -34.35 & -5.36 \\
9 & $\mathbf{2 i}$ & 42.23 & -45.36 & -6.25 \\
10 & $\mathbf{2 j}$ & 36.17 & -27.83 & -5.26 \\
11 & $\mathbf{2}$ & 40.07 & -28.99 & -5.37 \\
12 & $\mathbf{2 l}$ & 38.11 & -43.51 & -6.62 \\
13 & $\mathbf{2 m}$ & 42.44 & -40.56 & -6.18 \\
14 & $\mathbf{2 n}$ & 45.36 & -37.47 & -6.12 \\
15 & $\mathbf{2 o}$ & 41.02 & -37.78 & -5.89 \\
16 & Citalopram & 48.32 & -46.56 & -6.93 \\
\hline
\end{tabular}

and -0.18 , respectively resemble citalopram with a score of -0.26 . However, the drug-likeness scores of $\mathbf{2 e}, \mathbf{2 f}$ and $\mathbf{2 g}$ do not parallel strictly with their in vivo biological activites.

\subsection{Docking studies}

The level of antidepressant activity of compounds (2a2o) was studied by in silico modelling techniques by automated docking of ligands to the serotonin binding site of arabinase (table 5). ${ }^{17}$

The docking studies revealed that compounds $\mathbf{2 b}, \mathbf{2 c}$, $\mathbf{2 e}, \mathbf{2 g}, \mathbf{2 i}, \mathbf{2} \mathbf{l}$ and $\mathbf{2 m}$ have the highest negative glide score ranging from -45 to -40 (citalopram -46.56 ). These compounds are expected to form more stable drug-receptor complexes. However, these were less active than compound $\mathbf{2 f}$ with a score of -36.22 . All the compounds were analysed for hydrophobic and Vanderwaals interactions, since very few compounds were found to exhibit hydrogen bonding with the receptor. Other compounds were found to exhibit no hydrogen bonding with the receptor.

\section{Conclusion}

The present work led to the discovery of antidepressant molecules containing 1,3 dihydroisobenzofuran (phthalan) pharmacophore of the reputed drug, citalopram, but with far less structural embellishments. Antidepressant activity was evaluated for all the proposed compounds by using forced swim test and tail suspension test. Compound $2 \mathbf{f}$ exhibited potent activity than the reference drug, citalopram. Other compounds showed moderate to good antidepressant activity. The synthesized com- pounds showed antidepressant activity without having the usual aminoalkyl side chain characteristic of CNS active molecule, citalopram.

\section{Supplementary information}

The electronic supporting information can be seen in www.ias.ac.in/chemsci.

\section{Acknowledgement}

$\mathrm{CP}$ thanks the Council of Scientific and Industrial Research (CSIR), New Delhi, India for the research fellowship. IC thanks Dr. KV Raman, Dean, Mother Theresa Post Graduate and Research Institute of Health Sciences, Puducherry, India. KSK thanks Prof. P P Mathur, Head, Bioinformatics Centre, Pondicherry University, Puducherry, India.

\section{References}

1. Wong M L and Licinio J 2001 Nat. Rev. Neurosci. 2343

2. Little A 2009 Am. Fam. Physician 80167

3. (a) Richard C and Shelton M D 2003 J. Clin. Psychiatry 5 27; (b) Koenig A M and Thase M E 2009 Pol. Arch. Med. Wewn. 119 478; (c) Inoue T, Kusumi I and Yoshioka M 2002 Curr. Drug Targets: CNS Neurol. Disord. 1519

4. Stahl S M 2006 Essential psychopharmacology: The prescriber's guide (New York: Cambridge University Press)

5. Berton O and Nestler E J 2006 Nat. Rev. Neurosci. 7137

6. (a) Zhu W, Ma S, Qu R and Kang D 2006 Life Sci. 79 74; (b) Petersen P V, Lassen N, Hansen V, Huld T, Hjortkjaer J, Holmblad J, Nielsen I M, Nymar M, 
Pedersen V, Jorgensen A and Hougs W 1966 Acta Pharmacol. Toxicol. 24 121; (c) Petersen P V, Lassen N, Norgaard J and Huld T 1969 U.S. Patent 345765; (d) Bigler A J, Bogeso K P, Toft A and Hansen V 1977 Eur. J. Med. Chem. 12289

7. Feighner J P 1999 J. Clin. Psychiatry (supp. 4) 604

8. Briner K and Dodel R C 1998 Curr. Pharm. Des. 4291

9. Labbate L A, Grimes J B and Arana G W 1998 Biol. Psychiatry 43904

10. Ghosh M N 2005 Fundamentals of experimental pharmacology, 3rd Edition (Hilton and company, Kolkatta) pp. 190-197

11. Porsolt R D, Bertin A and Jalfre M 1977 Arch. Int. Pharmacodyn. Ther. 229327

12. (a) Steru L, Chermat R, Thierry B and Simon P 1985 Psychopharmacology (Berl) 85 367; (b) Nielsen D M, Carey G J and Gold L H 2004 Eur. J. Pharmacol. 499 135

13. (a) Praveen C, Sagayaraj Y W and Perumal P T 2009 Tetrahedron Lett. 50 644; (b) Praveen C, Kiruthiga P and
Perumal P T 2009 Synlett. 1990; (c) Praveen C, Karthikeyan K and Perumal P T 2009 Tetrahedron 65 9244; (d) Praveen C, Jegatheesan S and Perumal P T 2009 Synlett. 2795; (e) Praveen C, Kalyanasundaram A and Perumal P T 2010 Synlett. 777; (f) Praveen C, Kumar K H, Muralidharan D and Perumal P T 2008 Tetrahedron 64 2369; (g) Praveen C, Parthasarathy K and Perumal P T 2010 Synlett. 1635. (h) Praveen C, Iyyappan C and Perumal P T 2010 Tetrahedron Lett. 51 4767; (i) Praveen C, Dheenkumar P and Perumal P T 2010 Bioorg. Med. Chem. Lett. 20 7292; (j) Praveen C and Perumal P T 2011 Synlett. 521; (k) Praveen C, Ayyanar A and Perumal P T 2011 Bioorg. Med. Chem. Lett. 214170

14. Hiroya K, Jouke R, Kameda M, Yasuhara A and Sakamoto T 2001 Tetrahedron 579697

15. Lipinski C A, Lombardo F, Dominy B W and Feeney P J 2001 Adv. Drug Deliv. Rev. 463

16. Tetko I V 2005 Drug Discov. Today 101497

17. We didn't make any homology modelling for the receptor serotonin (5-HT) 